\title{
THE CANTILEVER BEAMS ANALYSIS BY THE MEANS OF THE FIRST-ORDER SHEAR DEFORMATION AND THE EULER-BERNOULLI THEORY
}

\author{
Dino KLJUČANIN, Abaz MANĐUKA
}

\begin{abstract}
The effect of the Timoshenko theory and the Euler-Bernoulli theory are investigated in this paper through numerical and analytical analyses. The investigation was required to obtain the optimized position of the pipes support. The Timoshenko beam theory or the first order shear deformation theory was used regarding thick beams where the shearing effect of the beam is considered. The study of the thin beams was performed with the Euler-Bernoulli theory. The analysis was done for stainless steel AISI-440C beams with the rectangular cross-section. The steel beams were a cantilever and stressed under varying point-centred load.
\end{abstract}

Keywords: AISI-440C; Cantilever; Euler-Bernoulli beam theory; First order shear deformation theory; Timoshenko beam theory

\section{INTRODUCTION}

The importance of beam theories applications can be seen in many engineering fields. Their use comprises applications in machine design, mechatronics, the design of components, etc. These theories are used in the analysis of thin and thick beams. The thick beam theory was introduced by Timoshenko. It is based on shear deformation that takes place due to the bending of the beam.

In order to optimize design and to obtain the most precise results, various cases of the pipes supports were examined. The piping requires various location positioning due to terrain specification. In order to minimize the required materials and to achieve the best aesthetic, the behaviour of end pinned beams is investigated. The examination is done through numerical and analytical methods. One of the mistakes that is made in practice is neglecting of the shearing component in the material that happens in certain cases regarding the beam geometry. For FEM analysis, one of the latest numerical algorithms will be used (NASTRAN). This paper will determine the optimal positioning of the beam and present the comparison of the results obtained through analytical and numerical analyses. In order to obtain the best results and avoid neglecting of the aforementioned shearing component of this type of beams, the Euler-Bernoulli and Timoshenko Beam theories are applied in certain cases.

The Euler-Bernoulli theory has been successfully applied in engineering practice. This theory served as the base to formulate a theory for the finite displacement in the beam and post-buckling analysis [1]. Ghosh used this theory as a base for developing and implementing a solution for shape memory polymers [2]. Xue and Khawaja successfully applied the Euler-Bernoulli theory in the analytical study of sandwich structures [3]. The Euler-Bernoulli theory in fractional form is successfully used in the experimental analysis for micro-beams [4].

Labuschagne et al. have shown analysis of the EulerBernoulli and Timoshenko beam theories alongside with two-dimensional elasticity theory where the Timoshenko theory application had the most desirable results [5]. In the numerical and analytical study, the Timoshenko shear deformation theory was used as a foundation for the investigation of the vibrational performances in continuous beam [6]. For bending analysis of the cantilever isotropic beam, this theory was utilized as a base for the development of the theory for the displacements and stress [7]. Tessler et al. have done the refinement of the Timoshenko Beam theory for sandwich beams using Zigzag Kinematics [8]. The Timoshenko theory was used with Eringen nonlocal elasticity theory to form differential transformation method for the analysis of the thick nano-beams vibrations [9].

The accuracy of the Timoshenko theory is governed by the shear correction factor. Regardless of the shear correction factor three approaches i.e. two shear stress approaches and Saint-Venant are used for evaluation of shear correction factor [10]. Birman and Bert highlighted the importance of shear correction factor and evaluated its function for six methods in sandwich structures [11]. The effect of the different approaches used for shear coefficient can affect the accuracy in terms of natural frequencies [12].

\section{THICK AND THIN BEAM THEORIES}

The calculation of the observed values is done taking into consideration the stress caused by momentum made by the static force and the shear in the observed cross-section. The force is applied in the direction y and along the horizontal axis of the cantilever beam. A displacement occurring with respect to $x$ can be determined as Eq. (1):

$$
\frac{\mathrm{d} w}{\mathrm{~d} x}=\frac{P}{A \cdot G \cdot k}+\frac{\mathrm{d} \varphi}{\mathrm{d} x}
$$

Taking into consideration the angle of rotation which can be determined as Eq. (2)

$$
\frac{\mathrm{d} \varphi}{\mathrm{d} x}=\frac{P x}{E I}
$$


For the boundary condition for the value of $x_{1}$ and $x_{2}$, this equation can be expressed as Eq. (3) and Eq. (4), or in the final form given with Eq. (5):

$\mathrm{d} \varphi=\int_{x_{2}}^{x_{1}} \frac{P x}{E I} \mathrm{~d} x$

$\varphi(x)=\frac{P}{E I} \int_{x_{2}}^{x_{1}} x \mathrm{~d} x$

$\varphi(x)=\frac{1}{2}\left(\frac{P x_{1}^{2}}{E I}-\frac{P x_{2}^{2}}{E I}\right)$

After the integration is finished, and if the maximum distance of the beam is denoted as $l$, the final term for the angle of rotation with respect to $x$ will have the form expressed with Eq. (6):

$\varphi(x)=\frac{1}{2}\left(\frac{P l^{2}}{E I}-\frac{P x^{2}}{E I}\right)$

After all conditions for integration are obtained, the main equation can be written as Eq. (7):

$w(x)=\int_{x}^{l} \frac{P}{A \cdot G \cdot k} \mathrm{~d} x+\frac{1}{2} \int_{x}^{l}\left(\frac{P l^{2}}{E I}-\frac{P x^{2}}{E I}\right) \mathrm{d} x$

Integration with respect to $\mathrm{d} x$ will transform the aforementioned equations to final forms Eq. (8) and Eq. (9):

$$
\begin{aligned}
& w(x)=\frac{P}{A \cdot G \cdot k}(l-x)+\frac{P}{6 E I}\left(2 l^{3}-3 l^{2} x+x^{3}\right) \\
& w(x)=P\left(\frac{(l-x)}{A \cdot G \cdot k}+\frac{\left(2 l^{3}-3 l^{2} x+x^{3}\right)}{E I}\right)
\end{aligned}
$$

Where: $E$ - Young's modulus of elasticity (GPa); $G-$ Shearing modulus (MPa); $K$ - Timoshenko coefficient (-); $I$ - Inertia moment $\left(\mathrm{mm}^{4}\right) ; A$ - The cross-section of the observed beam $\left(\mathrm{mm}^{2}\right) ; P$ - Force acting on the element $(\mathrm{N})$.

The phenomenon of the thick beams was developed by Timoshenko and this theory was named after him. The principle of this theory is in the fact that beams that are considered thick, or that are of length twenty times shorter than their thickness, perform differentially from thin beams. The basic assumption is predicted by the Euler-Bernoulli theory, which states that sections of the beam plane remain perpendicular to the longitudinal direction.

The deformation which happens due to bending of the beam can only be correct for the long and thin beams. In the case of thick beams, shear deformation must be taken into consideration. In this study, the force was pointed in the following distances $130 \mathrm{~mm}, 260 \mathrm{~mm}, 390 \mathrm{~mm}, 520 \mathrm{~mm}$ and at the end, i.e. $650 \mathrm{~mm}$ from the fixed support. The end values of the applied forces are shown in Tab. 1.

Table 1 Values of the applied force

\begin{tabular}{|c|c|c|c|c|}
\hline F1 & F2 & F3 & F4 & F5 \\
\hline$(\mathrm{N})$ & $(\mathrm{N})$ & $(\mathrm{N})$ & $(\mathrm{N})$ & $(\mathrm{N})$ \\
\hline 4414.5 & 3433.5 & 2452.5 & 1471.5 & 882.9 \\
\hline
\end{tabular}

\section{NUMERICAL SIMULATION}

Analysis was based on stainless steel beams with squared-rectangular cross-sections, as it is shown in Fig. 1. Used steels have many advantages compared to other commercial steels. Due to their high chromium content, they have good corrosion resistance. These steels are also used in applications such as medical equipment, kitchen hardware, transport systems of fluids, and structural parts. As a structural part, these steels are widely used due to their ability to sustain harsh outdoor conditions without any maintenance.

All beams were made of stainless structural steel with properties shown in Tab. 2. In order to obtain results for this type of load by FEM, NASTRAN algorithm was used. This algorithm was developed by NASA (NASA Stress Analysis) and was used as an addition to Autodesk Inventor stress analysis tools.

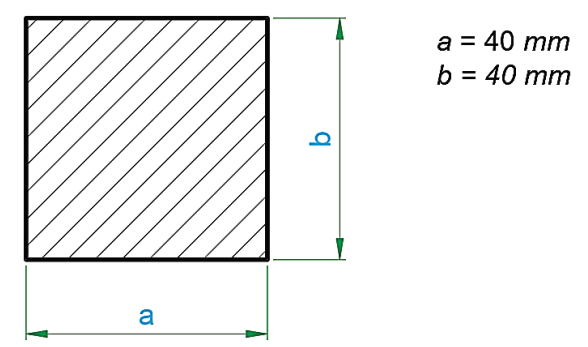

Figure 1 Cross section of the examined beams

The meshing setup was made for $15 \mathrm{~mm}$ element size as it is shown in Fig. 2. In order to increase the accuracy of performed analysis, parabolic element order was used. The cross-section of the beam was quadratic with dimensions of $40 \times 40 \mathrm{~mm}$. The Timoshenko theory was applied on the beam with a length of $650 \mathrm{~mm}$, while the Euler-Bernoulli theory was applied to the beam with a length of $950 \mathrm{~mm}$.

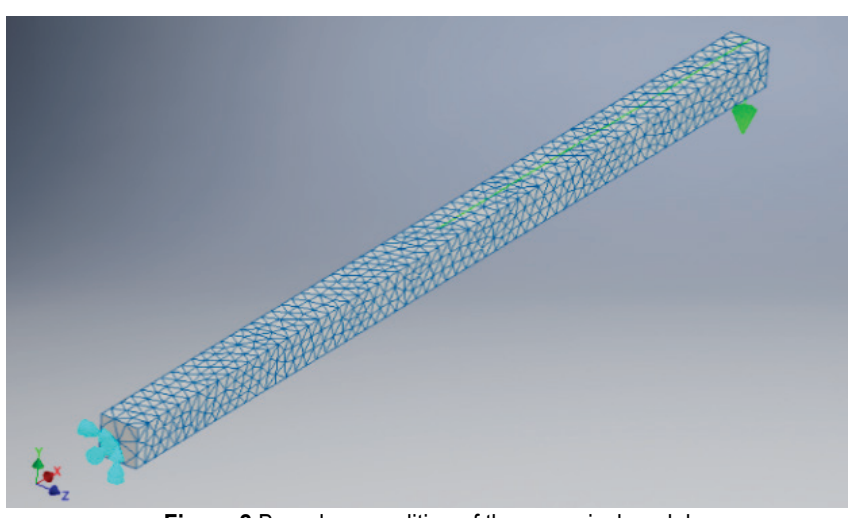

Figure 2 Boundary condition of the numerical model 
Table 2 Properties of the used material - AISI 440C

\begin{tabular}{|c|c|c|c|c|}
\hline Designation & $E$ & $G$ & $n$ & $K$ \\
\hline Unit & $(\mathrm{GPa})$ & $(\mathrm{MPa})$ & - & - \\
\hline Value & 206.7 & 83900 & 0.27 & 0.833 \\
\hline
\end{tabular}

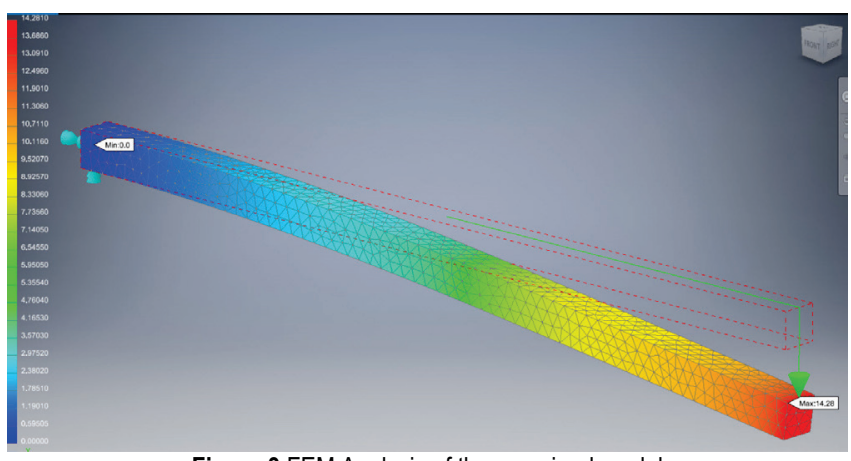

Figure 3 FEM Analysis of the examined model

Investigation of the non-supported cantilever beams included a fixed setup of the support (Fig. 2.) with no translation and rotation as the boundary condition and remote force according to the specified length in the $x, y, z$ coordinate system. A detail from the experimental run is shown in Fig. 3 .

\section{RESULTS EVALUATION - ANALYSIS COMPARISON}

Nastran module was used as an add-in module in Autodesk Inventor stress analysis environment. The results were obtained for each case that consisted of various distances at which the values of the aforementioned forces were applied. The analytical results in the case of the Timoshenko theory obtained for all the distances of the applied forces are shown in Tab. 3 .

The analytical analysis and calculation of the second beam displacement with the length of $950 \mathrm{~mm}$, were done by means of the Euler-Bernoulli theory and the results for all the examined cases of applied forces are shown in Tab. 4.

Table 3 Analytical results for the first beam

\begin{tabular}{|c|c|c|c|c|c|c|}
\hline Case & Dist & $\mathrm{d} 1$ & $\mathrm{~d} 2$ & $\mathrm{~d} 3$ & $\mathrm{~d} 4$ & $\mathrm{~d} 5$ \\
\cline { 2 - 7 } No. & $(\mathrm{mm})$ & $(\mathrm{mm})$ & $(\mathrm{mm})$ & $(\mathrm{mm})$ & $(\mathrm{mm})$ & $(\mathrm{mm})$ \\
\hline 1 & 130 & 0.513208 & 0.399162 & 0.285116 & 0.171069 & 0.102642 \\
\hline 2 & 260 & 1.906193 & 1.482594 & 1.058996 & 0.635398 & 0.381239 \\
\hline 3 & 390 & 3.95901 & 3.07923 & 2.19945 & 1.31967 & 0.791802 \\
\hline 4 & 520 & 6.451715 & 5.018001 & 3.584286 & 2.150572 & 1.290343 \\
\hline 5 & 650 & 9.164365 & 7.127839 & 5.091314 & 3.054788 & 1.832873 \\
\hline
\end{tabular}

Table 4 Analytical results for the second beam

\begin{tabular}{|c|c|c|c|c|c|c|}
\hline $\begin{array}{c}\text { Case } \\
\text { No. }\end{array}$ & Dist. & $\mathrm{d} 1$ & $\mathrm{~d} 2$ & $\mathrm{~d} 3$ & $\mathrm{~d} 4$ & $\mathrm{~d} 5$ \\
\hline 1 & $(\mathrm{~mm})$ & $(\mathrm{mm})$ & $(\mathrm{mm})$ & $(\mathrm{mm})$ & $(\mathrm{mm})$ & $(\mathrm{mm})$ \\
\hline 2 & 380 & 1.602212 & 1.246165 & 0.890118 & 0.534071 & 0.320442 \\
\hline 3 & 570 & 12.35991072 & 4.628612 & 3.306151 & 1.983691 & 1.190214 \\
\hline 4 & 760 & 20.142091 & 15.613271 & 6.866622 & 4.119973 & 2.471984 \\
\hline 5 & 950 & 28.610924 & 22.252941 & 11.190050 & 6.714030 & 4.028418 \\
\hline
\end{tabular}

In the case of FEM simulation, the same conditions were applied in both cases of the end pinned beams. The aforementioned setup was used with respect to the real character of the point centred perpendicular load. The results obtained through numerical analysis are shown in Tab. 5.
Table 5 Numerical results for the first beam

\begin{tabular}{|c|c|c|c|c|c|c|}
\hline $\begin{array}{c}\text { Case } \\
\text { No. }\end{array}$ & Dist. & $\mathrm{d} 1$ & $\mathrm{~d} 2$ & $\mathrm{~d} 3$ & $\mathrm{~d} 4$ & $\mathrm{~d} 5$ \\
\hline 1 & 130 & 0.548650 & 0.427240 & 0.305140 & 0.182990 & 0.109720 \\
\hline 2 & 260 & 2.471700 & 1.923000 & 1.373500 & 0.824030 & 0.494350 \\
\hline 3 & 390 & 4.394800 & 3.418700 & 2.441800 & 1.465000 & 0.878910 \\
\hline 4 & 520 & 6.317400 & 4.913900 & 3.509900 & 2.106000 & 1.263500 \\
\hline 5 & 650 & 8.240500 & 6.409600 & 4.578100 & 2.746700 & 1.648100 \\
\hline
\end{tabular}

In order to show the detailed comparison of the beam theories in the case of thick beams, numerical analysis was performed for all the points of the applied loads with the same values and the results are shown in Tab. 6.

Table 6 Numerical results for the second beam

\begin{tabular}{|c|c|c|c|c|c|c|}
\hline Case & Dist. & $\mathrm{d} 1$ & $\mathrm{~d} 2$ & $\mathrm{~d} 3$ & $\mathrm{~d} 4$ & $\mathrm{~d} 5$ \\
\hline No. & $(\mathrm{mm})$ & $(\mathrm{mm})$ & $(\mathrm{mm})$ & $(\mathrm{mm})$ & $(\mathrm{mm})$ & $(\mathrm{mm})$ \\
\hline 1 & 190 & 1.709000 & 1.329400 & 0.949320 & 0.569210 & 0.341330 \\
\hline 2 & 380 & 7.709600 & 5.996200 & 4.282800 & 2.569300 & 1.541400 \\
\hline 3 & 570 & 13.710000 & 10.663000 & 7.616000 & 4.569200 & 2.741300 \\
\hline 4 & 760 & 19.708000 & 15.328000 & 10.949000 & 6.569300 & 3.941200 \\
\hline 5 & 950 & 25.708000 & 19.994000 & 14.281000 & 8.568600 & 5.141300 \\
\hline
\end{tabular}

The used methodologies are compared and standard errors according to the deviation, for the first case, are obtained and presented in Tab. 7. Margin error in the case of the first beam for all runs is presented in Tab. 8 .

Table 7 Standard Error for analytical and numerical method for the first beam

\begin{tabular}{|c|c|c|c|c|c|}
\hline Case & SE1 & SE2 & SE3 & SE4 & SE5 \\
\hline 1 & 0.017721 & 0.014039 & 0.010012 & 0.0059605 & 0.003539 \\
\hline 2 & 0.2827535 & 0.220203 & 0.157252 & 0.094316 & 0.0565555 \\
\hline 3 & 0.217895 & 0.169735 & 0.121175 & 0.072665 & 0.043554 \\
\hline 4 & 0.0671575 & 0.0520505 & 0.037193 & 0.022286 & 0.0134215 \\
\hline 5 & 0.4619325 & 0.3591195 & 0.256607 & 0.154044 & 0.0923865 \\
\hline
\end{tabular}

For the margin error at a confidence level of $90 \%$ and an alpha value of 0.1 , the standard error is calculated by means of Eq. (10) given as:

$$
S E=\frac{S D}{\sqrt{n}}
$$

Where: $S E$ - Standard Error: $S D$ - Standard Deviation; $n-$ number of examined values.

Table 8 Margin Error for analytical and numerical method for the first beam

\begin{tabular}{|c|c|c|c|c|c|}
\hline Case & ME1 & ME2 & ME3 & ME4 & ME5 \\
\hline 1 & 0.029151 & 0.023094 & 0.01647 & 0.009805 & 0.005822 \\
\hline 2 & 0.46513 & 0.362234 & 0.25868 & 0.15515 & 0.093034 \\
\hline 3 & 0.358437 & 0.279214 & 0.199333 & 0.119534 & 0.071646 \\
\hline 4 & 0.110474 & 0.085623 & 0.061182 & 0.03666 & 0.022078 \\
\hline 5 & 0.759879 & 0.590752 & 0.422119 & 0.253402 & 0.151976 \\
\hline
\end{tabular}

In the case of the first beam, two methodologies are compared and characteristic trend lines with corresponding equations are shown in Fig. 4.

In the case of the second beam at the confidence level of $90 \%$, using the same term given with Eq. (10), the standard error is determined and presented in Tab. 9.

Margin error for the second case with the critical value of 1.645 according to the $t$-test, as it was the case for the first beam, is obtained and presented by means of Tab. 10 . 


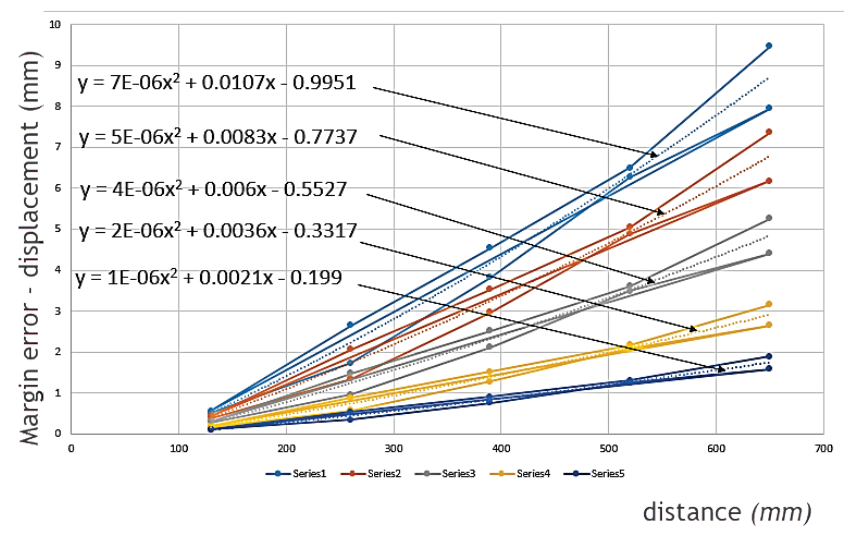

Figure 4 Comparison of the trend lines for analytical and numerical methods in the case of the first beam

Table 9 Standard Error for analytical and numerical method for the second beam

\begin{tabular}{|c|c|c|c|c|c|}
\hline Case & SE1 & SE2 & SE3 & SE4 & SE5 \\
\hline 1 & 0.053394 & 0.0416175 & 0.029601 & 0.0175695 & 0.010444 \\
\hline 2 & 0.879264 & 0.683794 & 0.4883245 & 0.2928045 & 0.175593 \\
\hline 3 & 0.6750405 & 0.5248645 & 0.374689 & 0.2246135 & 0.134658 \\
\hline 4 & 0.2170455 & 0.169035 & 0.120525 & 0.072365 & 0.043609 \\
\hline 5 & 1.451462 & 1.1294705 & 0.806979 & 0.4841875 & 0.2904425 \\
\hline
\end{tabular}

Table 10 Margin Error for analytical and numerical method for the second beam

\begin{tabular}{|c|c|c|c|c|c|}
\hline Case & ME1 & ME2 & ME3 & ME4 & ME5 \\
\hline 1 & 0.087833 & 0.068461 & 0.048694 & 0.028902 & 0.01718 \\
\hline 2 & 1.446389 & 1.124841 & 0.803294 & 0.481663 & 0.28885 \\
\hline 3 & 1.110442 & 0.863402 & 0.616363 & 0.369489 & 0.221512 \\
\hline 4 & 0.35704 & 0.278063 & 0.198264 & 0.11904 & 0.071737 \\
\hline 5 & 2.387655 & 1.857979 & 1.32748 & 0.796488 & 0.477778 \\
\hline
\end{tabular}

The comparison of two methodologies for the second beam is done and the trend lines are calculated and presented with corresponding equations for all the cases (Fig. 5).

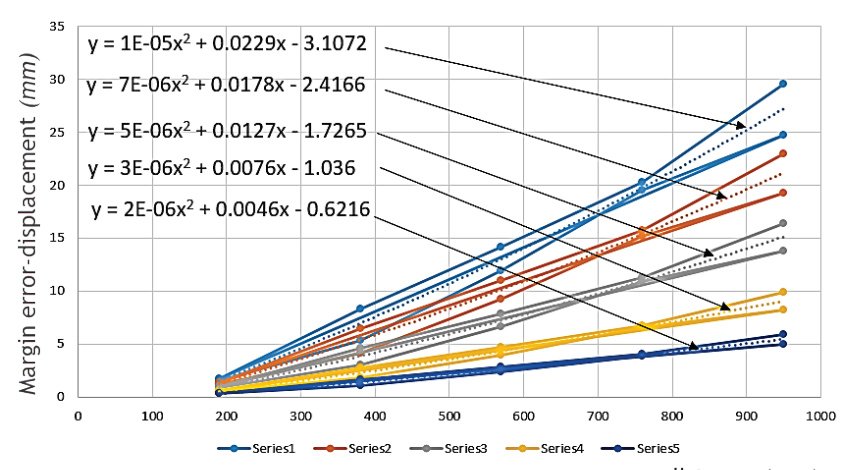

distance $(\mathrm{mm})$

Figure 5 Comparison of the trend lines for analytical and numerical methods in the case of second beam

\section{CROSS SECTION ANALYSIS AND ANALYSIS COMPARISON}

After the evaluation of the results, the differences between obtained displacements were present. The differences were asymmetrical. A certain cross-section of the support showed major differences for each magnitude of the force. The displacement deviations were present in the observed cross-section regardless of the load. The displacement deviations are shown in Fig. 6.

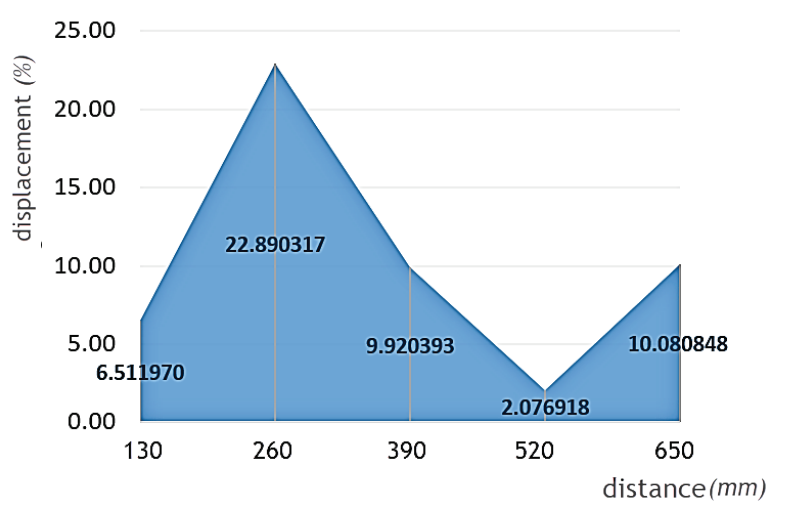

Figure 6 Comparison of the evaluated values for analytical and numerical method (shown in percentage) - Timoshenko theory

Compared results showed displacement deviations distinctions for both cases. The distinctions were the biggest in the first half part of the beam with up to a quartered deviation. Obtained results for the case of the Euler-Bernoulli theory are shown in Fig. 7.

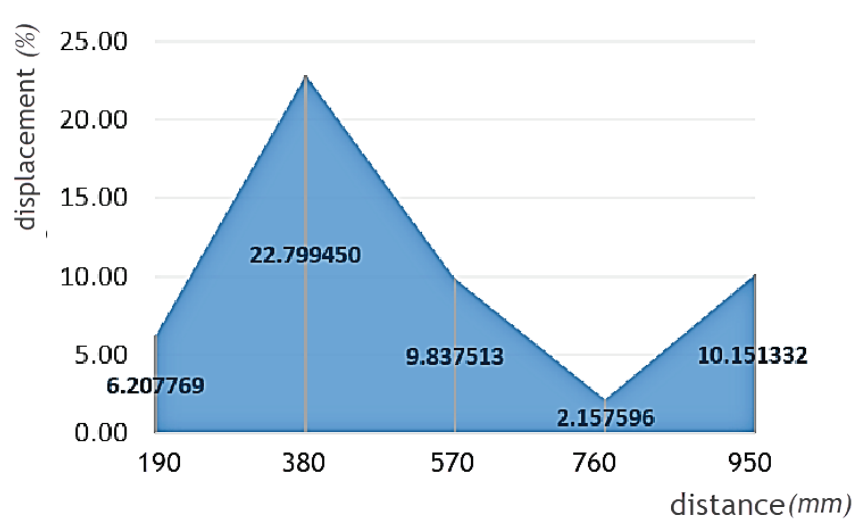

Figure 7 Comparison of the evaluated values for analytical and numerical method (shown in percentage) - Euler-Bernoulli theory

\section{DISPLACEMENT APPROXIMATION}

Due to different requirements in terms of the pipeline cross-sections, the combined mass of the transportation fluid and the pipes, it was necessary to investigate different values of the force applied. The investigations are done as a part of the development phase of the pipeline construction-setup to obtain the best values of the inclination angle that can provide the optimal fluid transportation. In the analysis of the optimal results for the two chosen supports numerical and analytical analyses were performed.

The numerical analysis showed the results obtained through calculation of analysed cases can be correlated and the relationship between the distance of the applied load and the displacement were linear, which can be seen from the diagram shown in Fig. 8.

The functional dependency for analytical solutions showed a slight deviation from the linear approximation of the displacement character in terms of distance of the load applied. The relation between them can be seen in Fig. 9. 


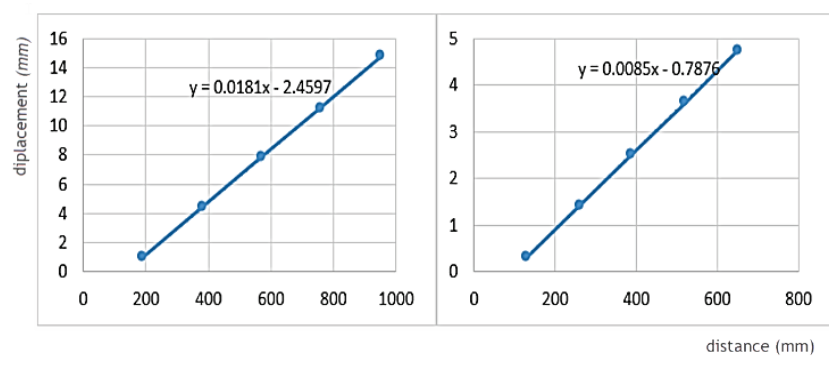

Figure 8 Distance-displacement relation preview - Numerical analysis

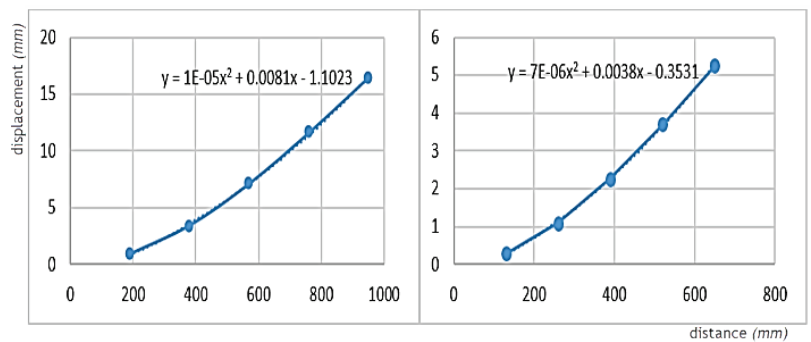

Figure 9 Distance-displacement relation preview - Analytical analysis

\section{CONCLUSION}

From the results obtained through numerical and analytical analyses, differences that were determined are noticeable and are in the sum range value of maximum $11 \%$ for the overall cross-section. The Timoshenko theory applied to thick beam analysis showed better overall fit compared to the Euler - Bernoulli theory. The displacement caused by the force that acted upon the different location on the beam had a linear character on all the evaluated beams.

Differences are more noticeable in the first part of the support and gradually elevated towards perpendicular midplane of the support. For the double value of the starting distance, the differences are most obvious with more than $20 \%$ for every tested case. The optimal results that provided the most reliable value regarding vertical displacement were the supports with the length of $520 \mathrm{~mm}$ and $760 \mathrm{~mm}$ for the first and second analysed beams, respectively. Future work is expected to be referenced regarding optimization and application of these theories in modified types of beams with a unique modified cross-section according to the design that is planned to be developed.

\section{REFERENCES}

[1] Usuki, T. (2008). A theory for the finite displacement of a thinwalled Bernoulli-Euler beam and lateral post-buckling analysis. Proceedings of the Royal Society of London A: Mathematical, Physical and Engineering Sciences, 1543-1570. https://doi.org/10.1098/rspa.2007.0256

[2] Gnoshl, P., Reddy, J. N., \& Srinivasa, A. R. (2013). Development and implementation of a beam theory model for shape memory polymers. International Journal of Solids and Structures, 50(3-4), 595-608. https://doi.org/10.1016/j.ijsolstr.2012.10.024

[3] Xue, H. \& Khawaja, H. (2016). Analytical Study of Sandwich Structures using Euler-Bernoulli Beam Equation. AIP Conference Proceedings: ICNPAA 2016 World Congress - $11^{\text {th }}$
International Conference on Mathematical Problems in Engineering, Aerospace and Sciences, La Rochelle.

[4] Sumelka, W., Blaszczyk, T., \& Liebold, C. (2015). Fractional Euler-Bernoulli beams: theory, numerical study and experimental validation. European Journal of Mechanics A/Solids, 54, 243-251. https://doi.org/10.1016/j.euromechsol.2015.07.002

[5] Labuschagne, A., v. Rensburg, N., \& v. d. Merwe, A. (2009). Comparison of linear beam theories. Mathematical and Computer Modelling, 49(1-2), 20-30. https://doi.org/10.1016/j.mcm.2008.06.006

[6] Zhang, X., Zhu, M., Wu, Z., \& Zhao, M. (2018). Dynamic Analysis of Timoshenko Beam with Arbitrary Constraints and a Further Optimization Based on Least Energy Principle. Mathematical Problems in Engineering, vol. 2018. https://doi.org/10.1155/2018/1269738

[7] Jadhav, V. A. \& Dahake, A. G. (2016). Bending Analysis of Deep Beam Using Refined Shear Deformation Theory. International Journal of Engineering Research, 5(3), 526-531.

[8] Tessler, A., Sciuva, M. D., \& Gherlone, M. (2007). Refinement of Timoshenko Beam Theory for Composite and Sandwich Beams Using Zigzag Kinematics, NASA Center for AeroSpace Information, Hampton.

[9] Ebrahimi, F. \& Nasirzadeh, P. (2015). A Nonlocal Timoshenko Beam Theory for Vibration Analysis of Thick Nanobeams Using Differential Transform Method. Journal of Theoretical and Applied Mechanics, 53(4), 1041-1052. https://doi.org/10.15632/jtam-pl.53.4.1041

[10] Marinetti, A. \& Oliveto, G. (2009). On the Evaluation of the Shear Correction Factors: a Boundary Element Approach.

[11] Birman, V. \& Bert, C. W. (2002). On the Choice of Shear Correction Factor in Sandwich Structures. Journal of Sandwich Structures and Materials, 4(1), 83-95. https://doi.org/10.1177/1099636202004001180

[12] Jensen, J. J. (1983). On the shear coefficient in Timoshenko's beam theory. Journal of Sound and Vibration, 7(4), 621-635. https://doi.org/10.1016/0022-460X(83)90511-4

\section{Authors' contacts:}

Dino KLJUČANIN, Engr.

University of Zenica

Fakultetska 1 ,

72000 Zenica, Bosnia and Herzegovina

kljucanin@live.com

\author{
Abaz MANĐUKA, PhD \\ University of Zenica \\ Fakultetska 1 , \\ 72000 Zenica, Bosnia and Herzegovina
}

